\title{
APPROXIMATION BY $(C, 1)$ AND ABEL-POISSON MEANS OF FOURIER SERIES ON HEXAGONAL DOMAINS
}

\section{Ali GuVEN}

Abstract. The approximation problems by Cesàro $(C, 1)$ means and by Abel-Poisson means of Fourier series on hexagonal domains are studied. The estimates for the rate of convergence of these means are obtained for functions in Lipschitz classes.

Mathematics subject classification (2010): 41A25, 42A10, 42B08.

Keywords and phrases: Hexagonal Fourier series, Cesàro means, Abel-Poisson means, Lipschitz class.

\section{REFERENCES}

[1] P. L. BUtZer, R. J. Nessel, Fourier analysis and approximation, Academic Press, 1971.

[2] R. A. DeVore, G. G. Lorentz, Constructive approximation, Springer-Verlag, 1993.

[3] B. Fuglede, Commuting self-adjoint partial differential operators and a group theoretic problem, J. Functional Analysis 16 (1974), 101-121.

[4] H. LI, J. Sun, Y. XU, Discrete Fourier analysis, cubature and interpolation on a hexagon and a triangle, SIAM J. Numer. Anal. 46 (2008), 1653-1681.

[5] J. Sun, Multivariate Fourier series over a class of non tensor-product partition domains, J. Comput. Math. 21 (2003), 53-62.

[6] Y. XU, Fourier series and approximation on hexagonal and triangular domains, Constr. Approx. 31 (2010), 115-138.

[7] A. Zygmund, Trigonometric series, Vol. I, Cambridge Univ. Press, 2nd edition, 1959. 